\title{
Cytotoxic lesion of corpus callosum in cerebral venous thrombosis-a case report
}

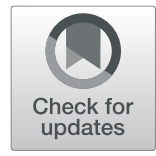

\author{
Tamaghna Ghosh', Simran Tanwar ${ }^{1}$, Shishir Chumber ${ }^{2}$ and Kavita Vani ${ }^{1 *}$ (D)
}

\begin{abstract}
Background: Veiled by a myriad of monikers, there has been a growing recognition of cytotoxic lesions in the splenium of the corpus callosum as a distinct clinical entity. Despite the varied nomenclature, they all describe restricting callosal lesions on diffusion weighted magnetic resonance imaging with near-complete reversibility on therapy. The currently accepted terminology for these lesions is cytotoxic lesions of the corpus callosum (CLOCC). Only one case of CLOCC associated with cerebral venous Thrombosis has been reported in literature to date.

Case presentation: While these lesions have most commonly been linked to antiepileptic drug therapy, we describe a case of a young adult who developed CLOCC in the background of cerebral venous thrombosis. We hypothesize that occlusion of the posterior pericallosal vein led to the lesion in the splenium. Early institution of anticoagulation therapy resulted in complete reversal of the abnormality, leading to full clinical recovery.

Conclusion: Cytotoxic lesions of the corpus callosum may rarely be associated with cerebral venous thrombosis. We emphasize the need for greater awareness, early imaging and aggressive therapy of this potentially curable entity. We further highlight cerebral venous thrombosis as a cause for CLOCC.
\end{abstract}

Keywords: Corpus callosum, Splenium, Cytotoxic lesion, Reversible splenial lesion syndrome, Cerebral venous thrombosis

\section{Background}

Transient lesions of the corpus callosum was first reported by Kim et al. in 1999 who noted the disappearance of focal lesions in the splenium of corpus callosum of two patients after withdrawing antiepileptic drugs [1]. Over the years, this entity has been variously described as mild encephalitis/encephalopathy with a reversible splenial lesion (MERS) [2] or reversible splenial lesion syndrome(RESLES) [3]. However the term cytotoxic lesions of the corpus callosum (CLOCC) is now considered most appropriate [4, 5]. CLOCC are secondary lesions associated with various entities including antiepileptics, encephalitis, gastrointestinal infections, metabolic disorders, malignancy, systemic lupus erythematosus, eclampsia, vitamin B12 deficiency and status migrainosus [5-7]. CLOCC in the setting of

\footnotetext{
* Correspondence: kavita2vani@gmail.com

'Department of Radiodiagnosis, RML, Delhi, India

Full list of author information is available at the end of the article
}

cerebral venous thrombosis (CVT) are however extremely rare. While there has been a previous report of CVT with concomitant CLOCC [8], a strong causal connection could not be established. We report a case of a young adult with CLOCC in the setting of CVT with no other predisposing factors. CVT is further implicated as the causal factor, as the CLOCC reversed with resolution of the CVT. We particularly wish to emphasize the need for early imaging and aggressive therapy in this potentially curable disease.

\section{Case presentation}

A 22-year-old male presented to the emergency room with complaints of severe, sudden onset, progressive bitemporal headache since the past 4 days associated with 4-5 episodes of projectile nonbilious vomiting. There was no history of fever, altered sensorium or neck stiffness. No previous co-morbidity was found and the patient was on no prior medications. On examination, 
he was found to have a GCS of 15 . There were no sensory or motor deficits and bilateral plantar response was flexor. Emergency NECT of the brain revealed illdefined hyperdensities extending along the course of the superior sagittal and straight sinus. Internal cerebral veins and the vein of Galen also appeared hyperdense (Fig. 1). Based on the suspicion of superior sagittal sinus thrombosis, the patient was admitted and MR venography was recommended. Subcutaneous injections of enoxaparin $0.6 \mathrm{ml}$ twice daily were started preemptively.

By the second day of admission, the patient complained of difficulty in getting out of bed and walking. Neurological examination revealed bilateral proximal lower limb weakness with grade $4 / 5$ power. There were, however, no sensory deficits, spasticity or hyperreflexia. The patient also complained of blurring of vision. Bedside ophthalmological examination failed to reveal any abnormalities.

MR examination showed hyperintensities replacing the normal flow void along the superior sagittal sinus on T1WI and T2WI. Subsequent MR venography revealed extensive partial filling defects in the superior sagittal sinus extending into the right transverse and sigmoid sinuses as well as the straight sinus and vein of Galen (Fig. 2a). There was also involvement of a few superficial cortical veins in the bilateral frontal lobes and left occipital lobe (Fig. 2c). High-resolution post-contrast T1 MPRAGE scan revealed a partial thrombus in the vein of Galen extending into the proximal part of the internal cerebral vein (Fig. 2f).

The study also revealed an isolated T2/FLAIR hyperintense ovoid midline lesion in the splenium. The lesion had high signal intensity on DWI imaging with corresponding low signal intensity on ADC mapping suggestive of diffusion restriction (Fig. 3). Post contrast imaging revealed an absence of enhancement of the lesion (Fig. 2b). The patient was put on warfarin $2 \mathrm{mg}$ once a day, in addition to the enoxaparin therapy. By day 6 , the patient had symptomatically improved enough to be discharged and was advised follow-up. Investigations into the cause of the cerebral venous thrombosis did not yield any answers. Work up for ANA titre, Protein C \& S, urinary protein excretion, lipid profile, and blood counts were all within the normal range.

Follow-up MRI scan with venography was done 3 weeks after the previous scan. It revealed complete disappearance of the splenial lesion without any sequelae (Fig. 4). MR venography revealed near complete resolution of the thrombus.

\section{Discussion}

CLOCC characteristically appear hyperintense on FLAIR and hypointense on T1-weighted imaging, show diffusion restriction, lack enhancement and tend to be relatively symmetric and in the midline $[3,5]$, all of which were found in our case.

Involvement of corpus callosum can occur in the following patterns:

A) Small ovoid to round lesion centred in splenium, as was seen in our case

B) Lesion in the splenium with extension into adjacent white matter

C) Lesion centred in the splenium with extension into the anterior portion of corpus callosum

These MRI findings regress spontaneously within weeks to months in most of the cases without any sequelae, differentiating it from ischaemia [5]. Complete disappearance of the splenial lesion was noted in our case in follow-up imaging done after 3 weeks.

CLOCC in the setting of cerebral venous thrombosis are rare. Liu et al in 2017 reported two cases of cerebral venous thrombosis with concurrent CLOCC in postpartum women who presented with headache and fever [8]. Left sided numbness and progressive mental

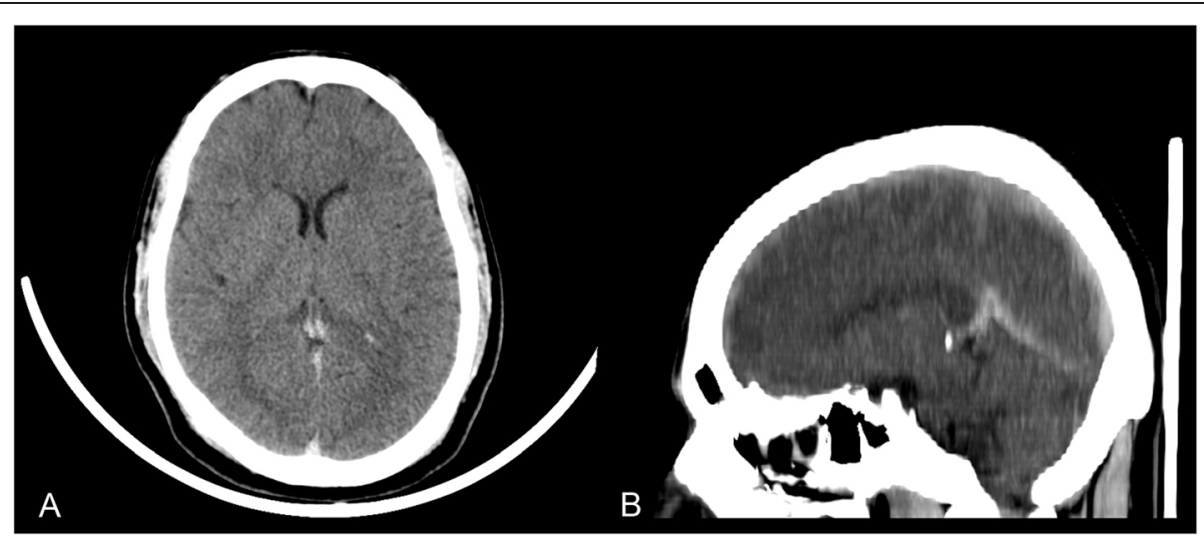

Fig. 1 Axial NECT showed 'dense clot' sign (a). Sagittal reconstruction revealed hyperdensity along the superior sagittal sinus and Straight sinus. 


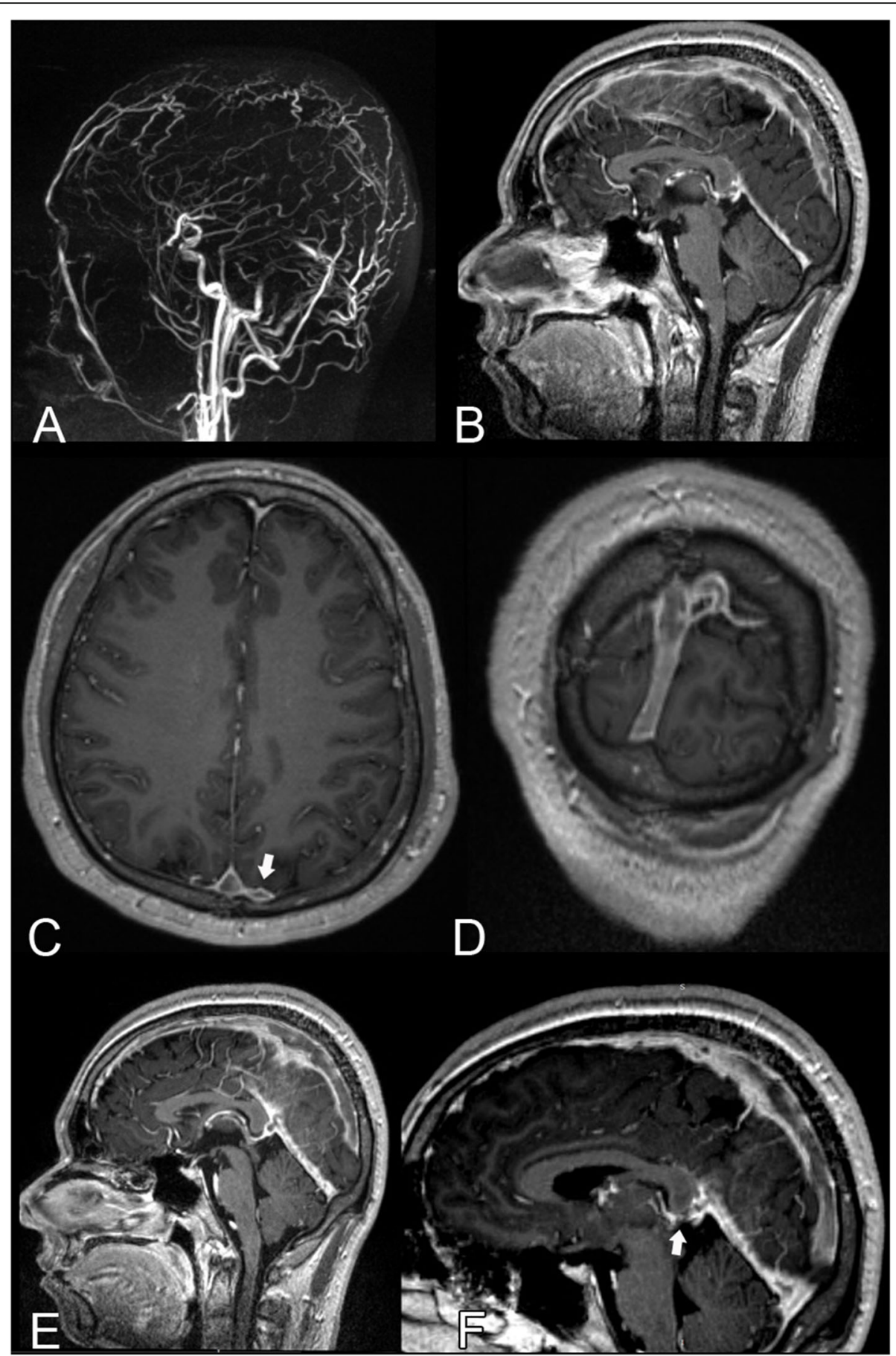

Fig. 2 Phase contrast MR venogram showed absence of flow along superior sagittal sinus, right transverse sinus and right sigmoid sinus (a). Contrast-enhanced MRI confirmed extensive cerebral venous sinus thrombosis (b). Associated thrombosis of the superficial cortical vein (arrow) seen in axial (c) and oblique-sagittal (d) post contrast T1 MPRAGE. Sagittal T1C+ images revealed partial thrombus in the vein of Galen (e) extending into the proximal part of the internal cerebral vein (arrow, $\mathbf{f}$.

deterioration was noted in one of the patient, not explained by the location of the lesion.

Clinical symptoms associated with CLOCC are variable and nonspecific reflecting a wide spectrum of underlying disease responsible for splenial abnormalities
[5, 9]. In our patient, the findings of weakness of bilateral lower limbs and gait ataxia were consistent with earlier studies which found them to be the second most common manifestation of splenial lesions [10]. The source of the visual disturbances could also be due to 


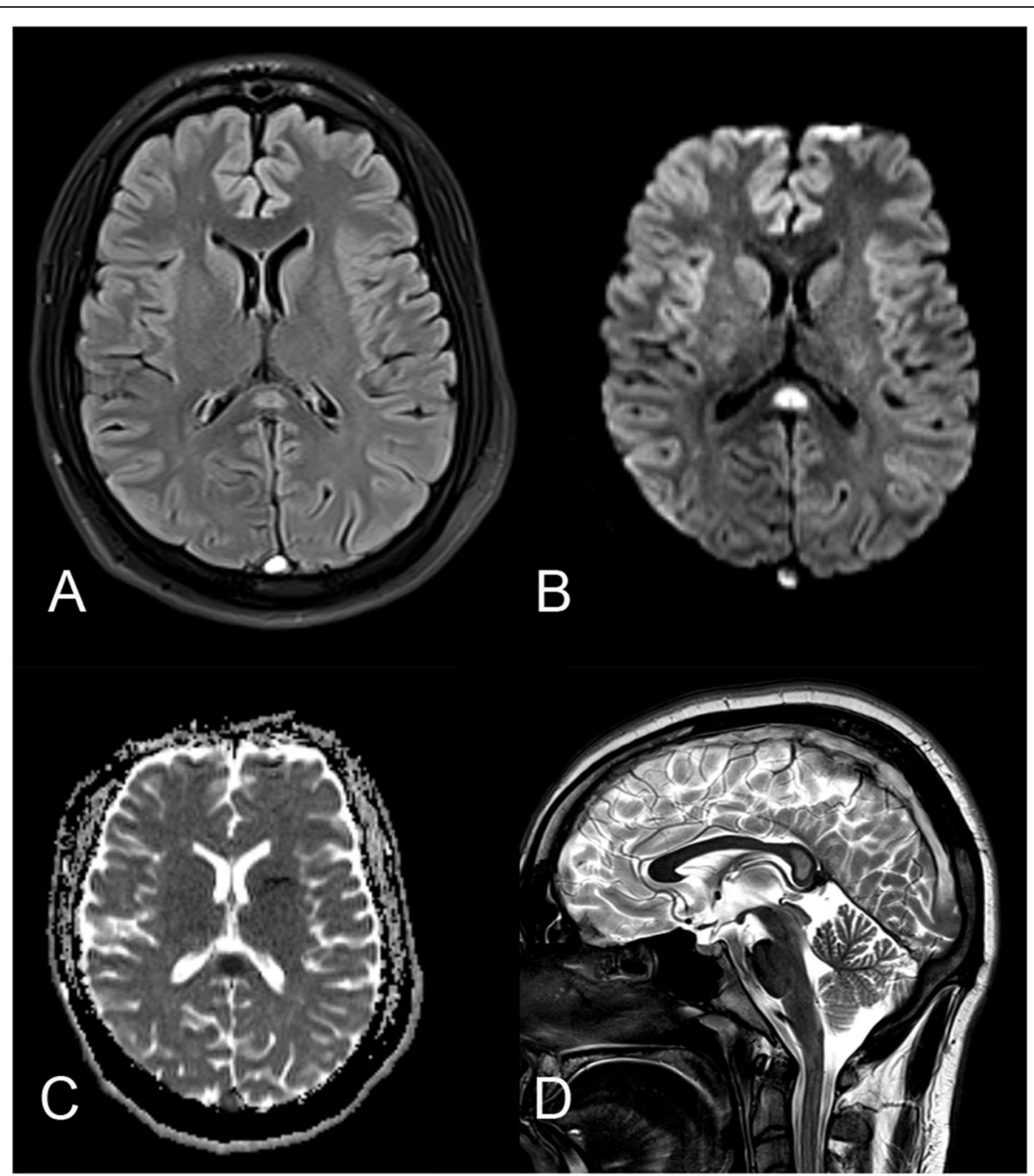

Fig. 3 Well-defined focal hyperintense lesion was seen in the splenium of the corpus callosum on axial T2/FLAIR (a) and sagittal T2 (d) images with corresponding area of restricted diffusion on DWI/ADC mapping (b, $\mathbf{c})$

involvement of the occipital visual fibres passing through the region $\mathrm{V}$ of the corpus callosum [11].

It is now known that both cytotoxic and vasogenic oedema occurs in setting of cerebral venous thrombosis $[12,13]$. Non-haemorrhagic parenchymal lesions showing restricted diffusion, in a setting of cerebral venous thrombosis, have been shown to be reversible [12]. Perfusion of the affected brain parenchyma is still possible after venous thrombosis if collateral pathways are present for drainage. Hence, the brain damage is often reversible, unlike in arterial thrombosis.

The CLOCC in our case was likely an outcome of the extensive cerebral vein thrombosis. The splenium of the corpus callosum is drained by short callosal veins and the posterior pericallosal vein. The posterior pericallosal vein shows considerable variation with possible drainage into the internal cerebral vein, the vein of Galen, the basal vein or even the posterior occipital vein $[14,15]$.
T1 MPRAGE images in our case, revealed a partially occlusive thrombus in the vein of Galen with extension into the internal cerebral vein. A possible explanation for the pathogenesis of the cytotoxic oedema could be the indirect occlusion of the posterior pericallosal vein by the partial thrombus in either the internal cerebral or the great cerebral vein. The reversibility of the lesion likely stemmed from the early resolution of the occlusive thrombus or presence of patent alternate drainage pathways via the short callosal vein or subependymal vein of the posterior third of corpus callosum.

Appenzeller et al. reported three cases of 'focal transient lesion' of SCC in active SLE patients [16]. Of the cases, one had additional cerebral venous thrombosis making the true aetiology of splenial lesion unclear. Literature search also revealed a case reported by Lai et al., of a postpartum woman with cerebral venous thrombosis presenting with severe headache [17]. The focal 


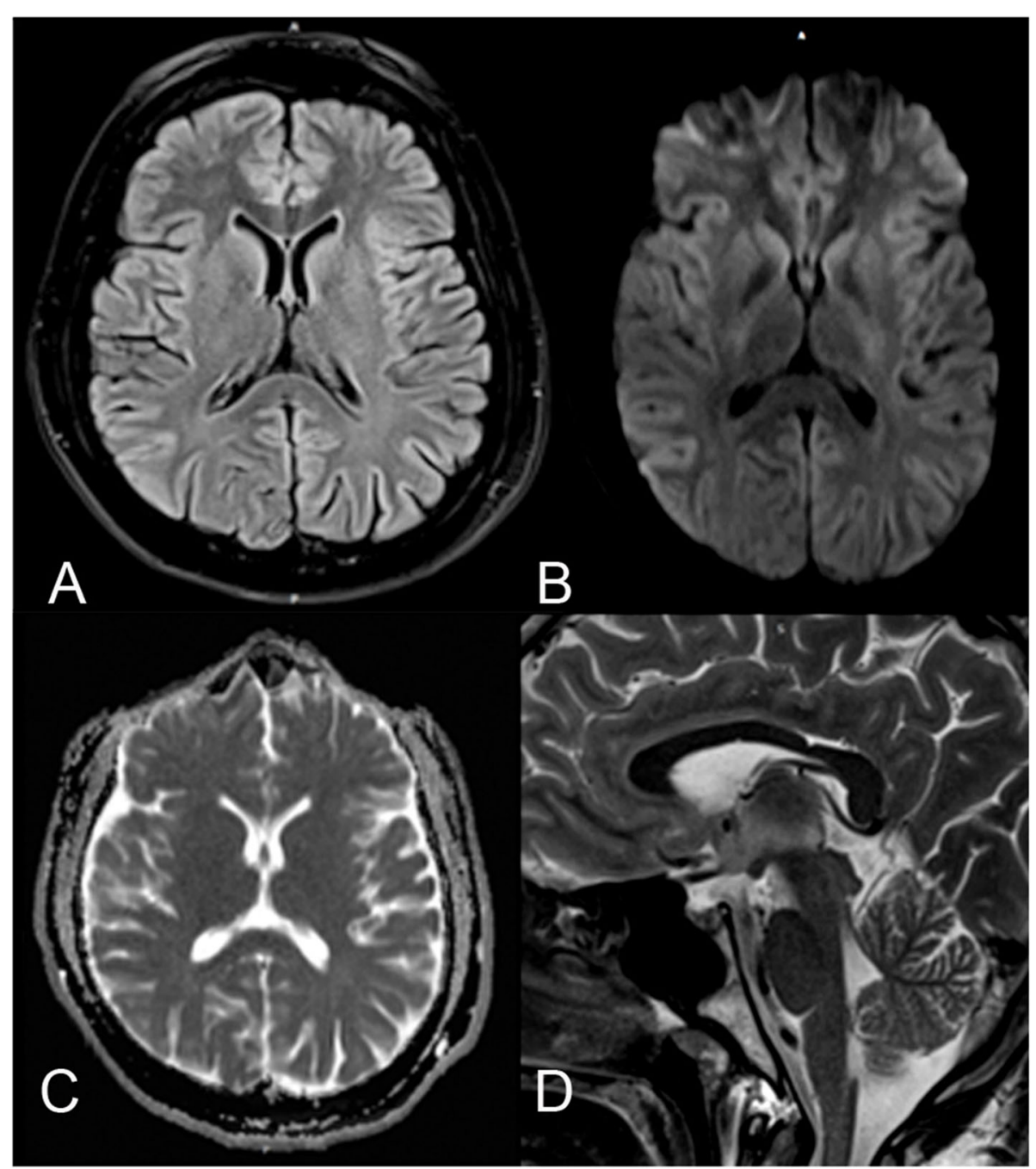

Fig. 4 Follow-up scan on day 21 after initial MR. Axial T2/FLAIR (a) and sagittal T2 (d) images showed total reversal of the splenial lesion. DWI/ ADC mapping confirmed complete resolution of the cytotoxic oedema $(\mathbf{b}, \mathbf{c})$

ovoid midline splenial lesion showing restricted diffusion was considered an 'infarct' and no follow-up imaging was done.

Our case lends credence to previous reports of transient cytotoxic lesions of splenium of the corpus callosum in the background of cerebral venous thrombosis.

\section{Conclusion}

DWI restricting lesions can develop in the corpus callosum due a variety of causes. A case of cytotoxic lesion in the splenium of the corpus callosum in the setting of cerebral venous thrombosis is described in a young adult. Comparison of MR imaging findings at initial presentation and on follow-up at 3 weeks leads us to hypothesize that the occlusion of the posterior pericallosal vein or its distributaries may lead to cytotoxic lesions in the splenium of the corpus callosum. Early institution of anticoagulation therapy has the potential to completely reverse the abnormality, leading to full clinical recovery.

\section{Abbreviations}

CLOCC: Cytotoxic lesions of corpus callosum; NECT: Non-enhanced computed tomography; CVT: Cerebral venous thrombosis; GCS: Glasgow Coma Scale; MR: Magnetic resonance (imaging); T1WI: T1-weighted image; T2WI: T2-weighted image; FLAIR: Fluid-attenuated inversion recovery; DWl: Diffusion-weighted imaging; ADC: Apparent diffusion coefficient: ANA: Antinuclear antibodies; MPRAGE: Magnetization prepared rapid gradient echo (MR sequence)

\section{Acknowledgements}

Not applicable

\section{Authors' contributions}

TG and ST collected and analysed the clinical data. TG and SC wrote the manuscript. KV guided, supervised and edited the manuscript. All authors read and approved the final manuscript.

Funding

None 


\section{Availability of data and materials}

YES. The datasets used and/or analysed during the current study are

available from the corresponding author on reasonable request.

Ethics approval and consent to participate

Not applicable.

\section{Consent for publication}

Written informed consent was obtained from the patient for publication of this case report and accompanying images.

\section{Competing interests}

The authors declare that they have no competing interests.

\section{Author details}

'Department of Radiodiagnosis, RML, Delhi, India. '2Department of Neurology, RML, Delhi, India.

Received: 19 November 2020 Accepted: 24 January 2021

Published online: 04 February 2021

\section{References}

1. Kim SS, Chang KH, Kim ST et al (1999) Focal lesion in the splenium of the corpus callosum in epileptic patients: antiepileptic drug toxicity? Am J Neuroradiol 20(1):125-129

2. Tada H, Takanashi J, Barkovich AJ et al (2004) Clinically mild encephalitis/ encephalopathy with a reversible splenial lesion. Neurology 63:1854-1858

3. Garcia-Monco JC, Cortina IE, Ferreira E et al (2011) Reversible splenial lesion syndrome (RESLES): what's in a name? J Neuroimaging 21:e1-e14

4. Galnares-Olalde JA, Vázquez-Mézquita AJ, Gómez-Garza G et al (2019) Cytotoxic lesions of the corpus callosum caused by thermogenic dietary supplements. Am J Neuroradiol 40(8):1304-1308

5. Starkey J, Kobayashi N, Numaguchi Y et al (2017) Cytotoxic lesions of the corpus callosum that show restricted diffusion: mechanisms, causes, and manifestations. Radiographics 37(2):562-576

6. Tetsuka S (2019) Reversible lesion in the splenium of the corpus callosum. Brain Behav 9(11):e01440

7. Maeda M, Tsukahara H, Terada H et al (2006) Reversible splenial lesion with restricted diffusion in a wide spectrum of diseases and conditions. J Neuroradiol 33:229-236

8. Liu J, Liu D, Yang B, Yan J et al (2017) Reversible splenial lesion syndrome (resles) coinciding with cerebral venous thrombosis: a report of two cases. Ther Adv Neurol Disord 10(12):375-379

9. Zhang S, Ma Y, Feng J (2015) Clinicoradiological spectrum of reversible splenial lesion syndrome (RESLES) in adults: a retrospective study of a rare entity. Medicine 94(6): 5512

10. Doherty MJ, Jayadev S, Watson NF et al (2005) Clinical implications of splenium magnetic resonance imaging signal changes. Arch Neurol 62(3):433-437

11. Hofer S, Frahm J (2006) Topography of the human corpus callosum revisited-comprehensive fiber tractography using diffusion tensor magnetic resonance imaging. Neuroimage 32(3):989-994

12. Forbes KP, Pipe JG, Heiserman JE (2001) Evidence for cytotoxic edema in the pathogenesis of cerebral venous infarction. Am J Neuroradiol 22:450-455

13. Ducreux D, Oppenheim C, Vandamme X (2001) Diffusion-weighted imaging patterns of brain damage associated with cerebral venous thrombosis. Am J Neuroradiol 22:261-268

14. Frerichs KU, Deckert M, Kempski O et al (1994) Cerebral sinus and venous thrombosis in rats induces long-term deficits in brain function and morphology —evidence for a cytotoxic genesis. J Cereb Blood Flow Metab 14(2):289-300. https://doi.org/10.1038/jcbfm.1994.36

15. Wolfram-Gabel R, Maillot C (1992) The venous vascularization of the corpus callosum in man. Surg Radiol Anat 14(1):17-21. https://doi.org/10.1007/ BF01628038

16. Appenzeller S, Faria A, Marini R (2006) Focal transient lesions of the corpus callosum in systemic lupus erythematosus. Clin Rheumatol 25:568-571

17. Lai W, Katirji B (2007) Splenium infarct due to cerebral venous thrombosis. Arch Neurol 64(10):1540

\section{Publisher's Note}

Springer Nature remains neutral with regard to jurisdictional claims in published maps and institutional affiliations.

\section{Submit your manuscript to a SpringerOpen ${ }^{\circ}$ journal and benefit from:}

- Convenient online submission

- Rigorous peer review

- Open access: articles freely available online

- High visibility within the field

- Retaining the copyright to your article

Submit your next manuscript at $\boldsymbol{\nabla}$ springeropen.com 